VOL. 44 (1991) [349-350]

\title{
Special subsets of the block sets of designs
}

\section{Ken Gray}

A combinatorial design is a way of choosing, from a given set of elements, a collection of subsets or blocks, satisfying certain properties. Such properties might include for instance, the size of the blocks, the number of blocks which contain each element, the intersection sizes of the blocks, and so on.

Substantial work has been done on the existence and classification of designs and the identification of their properties. Apart from their obvious pure mathematical interest, the search for designs with particular properties has been stimulated by their relevance to areas as diverse as the planning of experiments, coding theory and information security.

Investigation of the underlying properties of designs has in turn led to the investigation of special subsets of the sets of blocks of a design. Such subsets include, for example, supplementary difference sets, trades and the orbits of automorphisms. Also of interest are the subsets of blocks which have common intersection properties, contain a common element or have each element occurring in the same number of blocks.

Designs which have both the property that all the blocks have the same size and that every $t$-subset of the elements occurs in precisely the same number of blocks are known as $t$-designs. This thesis focuses on the special subsets of the block sets of such $t$-designs.

The first two chapters provide necessary background for the convenience of the reader. In Chapter 3, a construction analogous to that of C.J. Colbourn and M.J. Colbourn is developed from the special sets of starter blocks of cyclic and quasi-cyclic designs. The properties of starter blocks are also utilised in Chapters 4 and 5 , where the aim is to identify subdesigns, and hence partitions, of the $t$-designs carried by the blocks of the unique $[15,11,3]$ Hamming code; this follows on from work of E.P. Dawson. In these chapters the orbits of automorphisms are initially used to do this, but the consequential recognition of sets of blocks forming trades provides a viable alternative approach. Further, the use of trades also allows for construction of nonisomorphic designs and leads to consideration of block intersection and resolvability

Received 14th May 1991

Thesis submitted to The University of Queensland, December 1990. Approved March 1991. Supervisor Professor Anne Penfold Street.

Copyright Clearance Centre, Inc. Serial-fee code: 0004-9729/91 \$A2.00+0.00. 
properties. Trades carried by the general Hamming code and the relationship of the $[15,11,3]$ Hamming code to the Nordstrom-Robinson code are also investigated.

A full classification of all simple $(9,3,3)$ designs has been carried out by J.J. Harms, C.J. Colbourn and A.V. Ivanov. Chapter 6 focuses specifically on the resolvability of these designs, answering in this instance the question of whether an $m$-quasi-multiple of an affine plane is $m$-resolvable.

In the final three chapters, the notion of a $t-\left(v, k, \lambda_{t}\right)$ defining set of a design is introduced; this is defined to be a set of blocks of a design which can only belong to one $t$-design with the given parameters. It is shown that these defining sets have strong links to other types of subsets of the block sets of $t$-designs, in particular to trades, resolution classes and subdesigns. We also investigate their relationship to automorphism groups, to complementary designs, and to designs which are extensions or restrictions of other designs. Bounds on the number of blocks in a $t-\left(v, k, \lambda_{t}\right)$ defining set are established, with some particular families of designs being given particular consideration. Smallest defining sets of some small 2-designs and 3-designs are also determined.

As the properties of subsets are identified throughout this thesis, it becomes apparent that not only can different types of subsets be related in a natural way, but that the knowledge of a particular subset of the block set of a design will often disclose much useful information about the properties of the entire design.

Board of Secondary School Studies

PO Box 307

Spring Hill Qld 4004

Australia 